\title{
ON AN EQUATORIAL MOTION FOR TELESCOPES.
}

The object of the apparatus that forms the subject of the present paper, is to provide the means of giving a self-acting Equatorial Motion to an ordinary telescope, so as to enable the observer to keep any celestial body in the field of view, by a simple and inexpensive addition to the ordinary telescope and stand, and without requiring a previous knowledge of the position of the true meridian, or the latitude of the place of observation. The only method hitherto of accomplishing this object, has been by means of a permanent fixed axis, set parallel to the earth's axis, as is the case in fixed observatories, where both the position of the true meridian and the latitude of the place of observation have been previously ascertained; 
but with the apparatus about to be described, the preparation of the instrument occupies only a few minutes, and can be readily effected at the time by any observer.

This apparatus may consequently be not inappropriately called " the Portable Observatory," as it enables any one possessing an ordinary telescope mounted on a tripod stand to make a continuous observation of a celestial body, wherever he may happen to be, without having to touch the telescope after having once brought the object into the field of view.

The apparatus complete is before the meeting, and is also represented in the drawings, Plate 23, Figs. 1, 2, and 3.

In order to obtain an equatorial motion for the telescope, the first thing to be done is to set the axis about which it is to turn truly parallel to the earth's axis, because then any point in the axis of the telescope itself will move truly parallel to the equator, at whatever elevation the telescope may be set.

For this purpose, on an ordinary tripod stand A, Figs. 1 and 2, is rrounted a tube $B$, shown in section, in such a manner as to admit of both a horizontal and vertical angular motion, both of which can be firmly clamped in any position. In order to adjust the instrument suitably for the equatorial motion required, a small findertelescope $C$ is first inserted in the tube $B$, being made to fit it accurately by means of bell-metal collars fitting into corresponding bearings at the ends of the tube. The diaphragm which circumscribes the field of view of the finder has its centre, of course, in the axis of the finder; its diameter is $2^{\circ} 57^{\prime}$, being double the north polar distance of the Pole star; consequently the field of view of the finder, shown by the dotted lines in Fig. 2, takes in a circle exactly equal to that apparently described by the Pole star round the pole; and therefore, when the circle described by the Pole star coincides with the circumference of the field of view of the finder, the axis of the finder will be parallel to that of the earth.

In order to make the circle described by the Pole star coincide with the circumference of the field of view of the finder, a small brass sector or arm $\mathrm{D}$, shown in elevation in Fig. 3, is slipped over the finder, as shown in Fig. 2 , having two small holes iu it, and a 
narrow radial or longitudinal slit, which serves the purpose of an index. When two of the stars of the Great Bear constellation, namely, $\epsilon$ and $\zeta$ Urse Majoris, are seen through the two small holes of the sector, by the eye of the observer at $\mathrm{E}$, then the longitudinal slit or index points out that point of the edge of the field of view of the finder, to which the Pole star must be brought, by adjusting the finder by hand. This point will be apparently the one on the same side as the two stars $\epsilon$ and $\zeta$, since the position of the Pole star, which in reality is on the opposite side of the pole to the two stars above named, is inverted by the finder, which is a small ordinary astronomical telescope. When the finder is so adjusted that the Pole star is seen at the point thus indicated, at the same time that the two stars $\epsilon$ and $\zeta$ are seen through the two holes in the sector $D$, then the axis of the finder, and consequently of the tube $B$ encas ing it, is parallel to the earth's axis. The horizontal and vertical motions of the tube B are then clamped.

The accuracy of the adjustment may be tested by turning the finder $\mathrm{C}$ round its own axis, when the Pole star ought to remain stationary at the same point in the circumference of the field of view. If this be not the case, the adjustment must be corrected according to the deviation of the Pole star from a stationary position.

The finder $C$ with the sector $D$ is now removed, and the axis $F$ of the large telescope $G$ is inserted in the tube B, as shown in Fig. 1. The axis $\mathrm{F}$ is made to fit the tube accurately, in the same manner as the finder $\mathrm{C}$, by means of bell-metal collars fitting accurately into the corresponding bearings at the ends of the tube. Any point in the axis of the telescope will now move truly parallel to the equator, whatever inclination it may have to its axis $\mathrm{F}$; and consequently, when directed to any celestial body, it will truly follow the apparent course of that body, if turned round about the axis $\mathrm{F}$ at a fixed inclination and at a suitable uniform rate of motion.

To produce the required motion, the Right Ascension Circle or wheel $\mathrm{H}$ is fixed on the end of the axis $\mathrm{F}$; and underneath the stand $\mathrm{A}$ is placed a cylindrical pan of water $I$, containing the float $K$, the gradual descent of which, as the water runs out, communicates motion to the right ascension circle $\mathrm{H}$, by means of the cord $L$ passing over the pulley M. The 
water runs out of the pan $I$ through the syphon $N$, which is carried by the float $K$, so that the surface of the water is lowered at a uniform rate, the syphon descending with the water. This portion of the apparatus is, in fact, simply a revival of the ancient clepsydra.

The right ascension circle $\mathrm{H}$ has two grooves on its rim, the upper of which is exactly 24 inches in circumference, and the lower 25 inches nearly, these numbers being in the proportion of the sidereal to the mean lunar day. If the motion of the moon is to be observed, the cord $L$ is placed in the lower groove; if that of a star, in the upper; it is slightly tightened in the groove by means of a small clamp, and the loose end hangs by a plummet over a pulley fixed in one of the legs of the stand. The thickness of the cord should be twice the depth of the groores. The pulley $\mathrm{M}$ can be raised and lowered, so as to be always in the plane of the right ascension circle $H$, when the latitude of the place of observation is changed.

The pan $I$ is about $18 \frac{3}{4}$ inches in diameter; it contains $95 \frac{3}{4}$ pints in a depth of 12 inches. This quantity of water should be run off by the syphon $\mathrm{N}$ in 12 sidereal hours. The rate (which should be adjusted while the telescope is in motion) is determined by raising or lowering the syphon by means of a rack and pinion fixed to the float $K$. When adjusted, the syphon must deliver 1 gallon in 1 mean solar hour, or 1 pint in $7 \frac{1}{2}$ minutes.

When the syphon is first set in action, no motion will be imparted to the telescope, until the cord $\mathrm{L}$ is stretched sufficiently to overcome the friction of the collars of the axis $E$ in the tube $B$, and the inertia of the instrument. A stop-cock $\mathrm{O}$ is therefore provided, which is opened just at starting, thereby causing the float to sink rapidly; as soon as the body to be observed appears in the centre of the field of view, the stop-cock is closed, and then the action of the syphon alone gives sufficient motion to the telescope to cause the body to remain apparently stationary in the field of view.

As it is found that differences in the temperature of the water occasion some little variation of rate in the quantity delivered by the syphon, the rack and pinion should be graduated for such differences; or a selfregulating clepsydra might be constructed, either by using two cisterns and two syphons, one of the latter acting as a supply-pipe to the cistern 
containing the float connected with the telescope, in which case, since the difference in temperature affects both syphons alike, the difference in the rate of flow through them, which determines the rate of descent of the float, remuins constant; - or if one cistern only be used, by a thermometer carrying a piston attached to the syphon, the bulb of the thermometer being fixed underneath the float.

If the telescope be not well balanced about its centre of gravity, more or less weight will be required to move it, as its direction is varied, and consequently its motion will not be uniform. If, however, the float cover a large surface of water, and the syphon when adjusted for the mean temperature be immersed to some depth below the surface, no perceptible varjation of rate will be occasioned.

The action of the clepsydra produces a smoothness of motion not to be surpassed, and might be adapted with advantage to the requirements of an observatory, more especially as its rate may be so roadily brought under the immediate control of the observer.

It is to be remarked, that, independently of its application to the production of equatorial motion, the instrument above described, or more correctly the portion first described and exclusive of the clepsydra and of the parts connected with it, is designed to show a new and simple method by which an extended wire, the axis of a tube, or of a telescope, may be made parallel to the earth's axis, to a sufficient degree of accuracy to render it available for astronomical observations.

Other simpler forms might be adopted, by which one telescope only need be used, or a telescope and its finder; in the latter case, the finder having a field of view of $2^{\circ} 57^{\prime}$ would enable the observer to determine the polar axis, and might be so mounted as not to require to be removed: when the adjustment had been made. Where very powerful telescopes. are used, the smaller one, for finding the polar axis, might have a field of view of $3^{\circ}$, and the exact position of the Pole star within the circumference might be determined by a micrometer adapted for the purpose; this would admit of the requisite correction being made for refraction. Such a telescope, attached in the same manner as a finder to the present Polar axes in observatories, would serve to test the accuracy of their position ; while in Fraunhofer's form of Equatorial, (which is so arranged that the telescope will reach every part of the beavens without being 
interrupted by the framing or staud on which it is carricd,) it might be substituted for the Polar axis, whereby the optical axis of one telescope would be made the fixed axis, about which the other telescope would revolve.

Mr. Hopkrss exhibited his instrument to the meeting, and illustrated the method of adjustment, and mode of action.

The Chairman observed that it was undoubtedly a great advantage to be able to obtain a self-acting motion for an ordinary telescope, and by such as imple method as that proposed, the gradual descent of the water in the cylindrical pan, which was a beautiful mechanical contrivance for ohtaining a regular and steady motion; and the addition of the apparatus to the ordinary telescope would greatly extend its utility.

Mr. Hopkiss said he had found the instrument of great use in observations on the celestial bodies, and it answered the purpose intended very completely; the rate of the syphon was in practice set slightly slower than that of the stars, so that in case the observer left the telescope for a time, and on returning to it, found that the body he had been observing was no longer in the field of view, he would know that it must be in advance of the telescope, and he had simply to open the cock at the side of the pan, and run off a little more of the water, when the telescope would directly overtake the body, and the latter would again appear in the field. The two grooves in the right ascension circle enabled the observer to adapt his telescope to the moon or the stars, with the same adjustment of the syphon; if there were only one groove, the syphon would have to be readjusted separately for the moon and stars, which would be a troublesome process.

The Chairman asked whether a very large quantity of water would not be required, or a frequent filling up of the cistern, in the case of large instruments, in order to overcome the variable friction of the moving parts, and insure a steady motion; if this were required, the apparatus would then become inconveniently large.

Mr. Hopkins replied that where large telescopes were used, they might easily be so balanced about the axis of motion, as to render the 
friction to be overcome by the descending weight very nearly uniform. In the case of large instruments, he would also propose placing the syphon in a large detached tank, in any convenient protected situation, and connecting the water by a gutta-percha tube with the small pan attached to the telescope to impart the motion.

The Charrmas inquired whether difference in the temperature of the water did not affect the motion communicated to the telescope.

Mr. Hopkins stated that he had found by careful experiment that the differense of temperature had no pereeptible effect, when the bore of the syphon was more than $\frac{1}{4}$ inch in diameter; but when less than that, the difference did affect the rate of flow through the syphon. With a syphon 3-16ths inch bore, at $62^{\circ} \mathrm{Fahr}$, 1 pint of water flowed through in $15 \mathrm{l}$ seconds; but at $82^{\circ}$, the same quantity flowed through in 134 seconds; being a diminution of 17 seconds in the time, for an increase of $20^{\circ}$ in the temperature.

Mr. OsLer said he had seen the apparatus in operation on the previous day, and its action was very perfect and steady; he thought a simple and inexpensive equatorial motion was a great desideratum for ordinary telescopes; the clock-motion that was employed for the purpose was expensive, and beyond the reach of observers in general. This motion was generally adjusted slightly too fast, and was retarded byacheck-string held in the observer's hand, whenever required to bring the object into the field of view ; in Mr. Hopkins' apparatus, the adjustment was effected in the contrary way, by accelerating the motion when required by means of the regulating cock at the bottom of the pan. The objection most likely to be made to Mr. Hopkins' plan was that the water would freeze, when observations were made out of doors, as was most convenient inmany cases; but this might not prove any serious difficulty. In an observatory, the temperature might be kept up by artificial means, but this was objectionable, for the reason that the heated air, rising from the room, caused a flicker in the feld of view. He suggested that the difference in the velocity of flow through the syphon, under different temperatures, was due to the difference in the density of the water, the water becoming more fluid as its density was diminished by heat, and more viscid as it was increased by cold.

Mr. Hoprins said he had never experienced any difficulty from the freezing of the water, and in ordinary cases it was not likely an ob- 
server would remain at the instrument so long as would be requisite for the water to be cooled down to the freezing point; and even if it were desirable in severe weather to provide specially against this, it could be readily done by the addition of a little hot water in the pan, to raise the temperature at first; and the only inconvenience resulting would be the slight diminution in the rate of motion as the water cooled, which would be an error on the right side, as it would be instantly rectified by slightly opening the extru discharge cock at the bottom of the pan.

Mr. S. Lloyd, jun., suggested that salt water might be used in the pan, as less liable to freeze.

Mr. HoDge thought the apparatus was very ingenious, and a beautiful mode of giving a smooth regular motion; he did not think any difficulty from freezing need be apprehended, as various liquids might be employed, such as a mixture of alcohol and water, which would prevent the risk of freezing.

Mr. W. R. WILLs said he had had an opportunity of examining the instrument in operation, and had been much surprised and pleased with the perfect uniformity of motion communicated by the flow of the water; uniformity of movement having been obtained, he thought that the action of the machine would be smoother than that of any clock-motion. He had not had an opportunity of observing a star with the instrument, but had watched its steady and almost imperceptible motion in traversing across a stationary object.

The Chalrman remarked that it was essential that the body observed should appear quite stationary in the field of view, which could only be effected by securing a perfectly uniform and steady motion for the telescope, and this appeared to have been effectually aceomplished by Mr. Hopkins, and the simplicity of his apparatus was a great recommendation.

He proposed a vote of thanks to Mr. Hopkins, which was passed.

The Chatrman announced the presentation to the Institution Library of the Lowell Hydraulic Experiments, by Mr. James B. Francis, of Lowell, U. S. ; and of a large Drawing of the Pumping Engine of the Wolverhampton Water Works, by Mr. Henry Marten, of Wolverhampton.

The Meeting then terminated. 
EQUATORIAL TELESCOPE.

Plate 23.

Fig.1. Sectionat Elevation
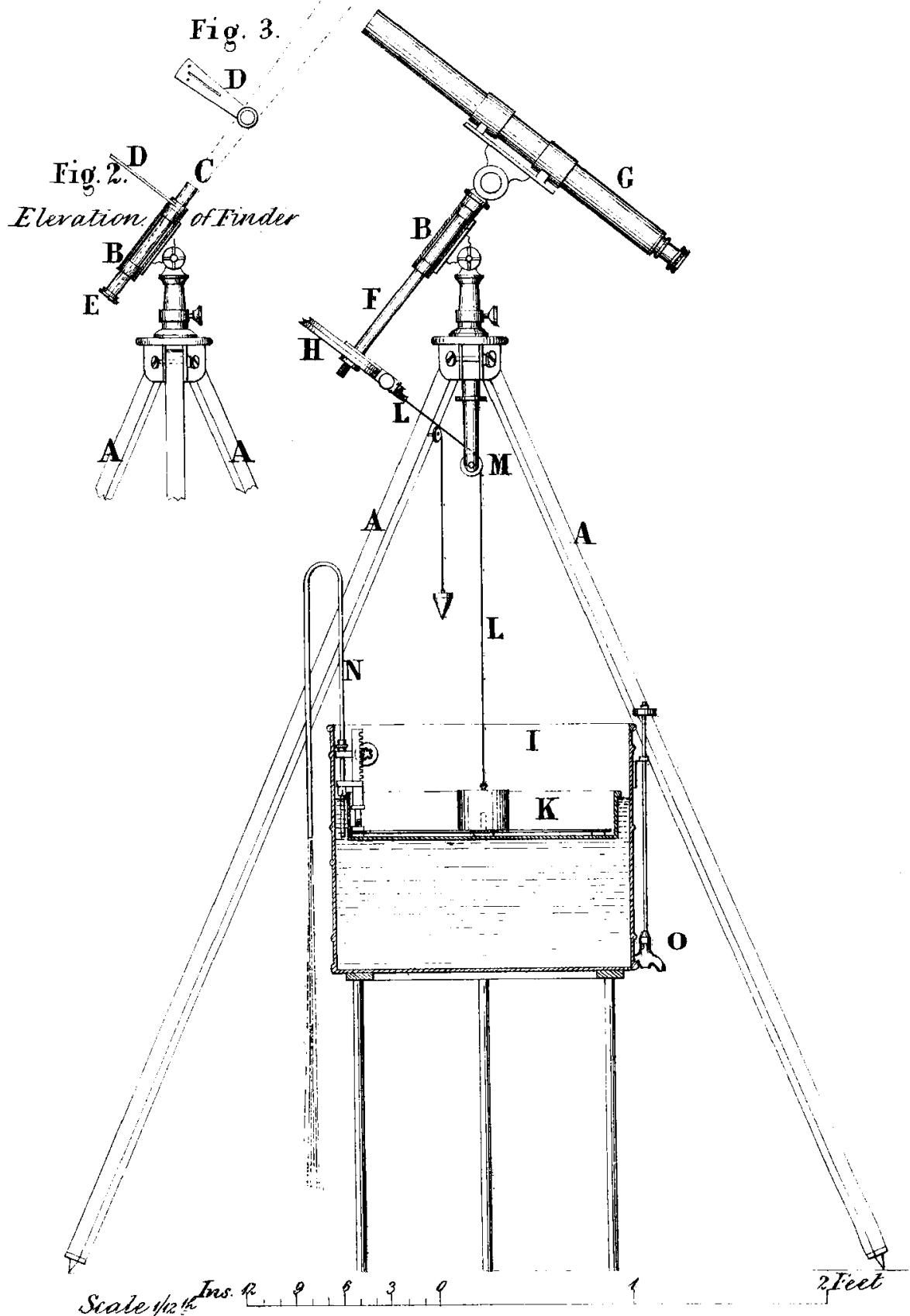

Proceedings trownloaded trom pme sagepub.com at UQ Library on June 4, 2016 